\title{
Aktuelle Strategien der Raucherentwöhnung
}

\author{
Current approaches to smoking cessation
}

\author{
Autoren \\ P. L. Bölcskei ${ }^{1}$ P. Davis-Wagner ${ }^{1}$ J. Grundnig ${ }^{2}$ P. Pommer ${ }^{2}$ \\ Institut \\ ${ }^{1}$ Institut für Raucherberatung \& Tabakentwöhnung, München \\ 2 Klinik am Kofel, Gesundheitszentrum Oberammergau
}

\section{Suchtmedizin}

Schlüsselwörter

Raucherentwöhnung

Abhängigkeit

kognitive Verhaltenstherapie

Nikotinersatztherapie

Ovareniclin

Bupropion

Keywords

smoking cessation

addiction

cognitive-behavioral therapy

nicotine substitution

Vareniclin

Bupropion eingereicht 28.08.2012

akzeptiert $\quad 06.02 .2013$

Bibliografie

DOI $10.1055 / \mathrm{s}-0032-1333053$

Dtsch Med Wochenschr 2013;

138: 902-907 - (c) Georg Thieme

Verlag KG · Stuttgart · New York ·

ISSN 0012-0472

\section{Korrespondenz}

Dr. med. Peter Pommer

Internist und Pneumologe,

Fachjournalist DFJV, Chefarzt

der Abteilung für Pneumologie,

Gesundheitszentrum

Oberammergau

Hubertusstr. 2

82487 Oberammergau

Tel. 08822/780

eMaildrpeterpommer@

googlemail.com

\section{Einleitung}

Tabakrauchen gehört zu den bedeutendsten Ursachen menschlicher Morbidität und Mortalität, obwohl prinzipiell komplett darauf verzichtet werden könnte. Der Versuch, Raucher zur Aufgabe dieses selbstschädigenden Verhaltens zu bewegen, ist daher grundsätzlich sinnvoll. Angehörige der Heilberufe und vor allem Ärzte aller Fachrichtungen tragen hier eine besondere Verantwortung. Untersuchungen haben gezeigt, dass selbst kurze motivierende ärztliche Gespräche messbare Erfolge haben $[35,36]$.

Diese Arbeit wendet sich an alle Ärzte mit direktem Patientenkontakt, in erster Linie an Ärzte in der Primärversorgung. Ziel ist es darzulegen, dass die Raucherentwöhnung ein Prozess ist, der trotz möglicher Rückschläge langfristig hohe Wirksamkeit hat. Auch Ärzte ohne besondere psychologische Ausbildung können in Kenntnis eines schrittweisen Entwöhnungsmodells mit geringem Aufwand ihren Patienten helfen. Ausrutscher bzw. Rückfälle sind dabei als Teil dieses Prozesses anzusehen und nicht als Scheitern. Bei ernsthafter Absicht und empathischer ärztlicher Begleitung ist der erfolgreiche Abschluss dieser stufenweisen Entwöhnung möglich.

Rauchen kann der Entspannung und dem Genuss dienen und der Geselligkeit zuträglich sein wichtige Werte im Lebenskonzept. Es kann wie ein Medikament gegen Nervosität, Stress, Angst und Depression wirken - nicht besonders langanhaltend, dafür mit einer innerhalb von Sekunden eintretenden Wirkung und niederschwelligem Zugang. Der Raucher spürt dadurch eine kurzzeitige Entlastung und einen Gewinn. Das Aufhören wird für den Raucher erst dann lohnenswert, wenn es zur Verwirklichung der eigenen Ziele wie Gesundheit, Selbstbestimmung und Wohlbefinden beiträgt.
Professionelle Raucherberater sind weit davon entfernt, Rauchen als Charakterschwäche zu sehen. Sie erkennen die Charakteristika einer möglichen Abhängigkeitserkrankung [40]. Hier hat der Hausarzt als Vertrauensperson mit regelmäßigem Kontakt zum Raucher besonders gute Voraussetzungen, einen Ausstiegsprozess zu fördern. Ärzten sollte bewusst sein, wie schwer jede ihrer Aussagen bei Patienten wiegt und wie wichtig motivierende Rückmeldungen für sie sind. Das bedeutet, rauchende Patienten empathisch in ihrem ambivalenten Denken wahrzunehmen und Zeit für ein positiv motivierendes Gespräch einzuplanen. Bei jedem Kontakt sollte erfragt werden, wie weit der Intentionsbildungsprozess vorangekommen ist, und Fortschritte sollten positiv verstärkt werden. Bei Rauchern, die noch nicht über das Aufhören nachdenken, gilt es, als ersten Schritt eine ambivalente Haltung zu fördern.

\section{Epidemiologie}

$\nabla$

Im Jahr 2000 wurden weltweit 5,5 Billiarden Zigaretten konsumiert [29]. Bei einer Weltbevölkerung von 6 Milliarden sind dies rund 1000 Zigaretten pro Kopf. 2002 errechnete die Weltgesundheitsorganisation (WHO) 4,8 Millionen durch Tabakrauchen bedingte Todesfälle [29]. Wenn es nicht gelingt, diesen Trend zu stoppen, ist zu erwarten, dass sich die Zahl der Todesopfer bis 2020 verdoppelt [21,30,38]. In diesem Jahrhundert sind 1 Milliarde Tote durch Rauchen zu erwarten. Dieselbe Zahl an Todesopfern wäre zu beklagen, wenn jede Stunde weltweit ein Jumbo-Jet abstürzen würde [17]. Laut WHO sterben $10 \%$ der erwachsenen Weltbevölkerung an den Folgen des Rauchens, die Hälfte aller Raucher wird aufgrund dessen erkranken [38]. Allein durch Passivrauchen sterben laut WHO jährlich rund 600000 Menschen, davon 165000 Kinder [39]. In Deutschland wird die Zahl der Todesopfer durch Passivrauchen jährlich auf 3330 geschätzt [24]. 


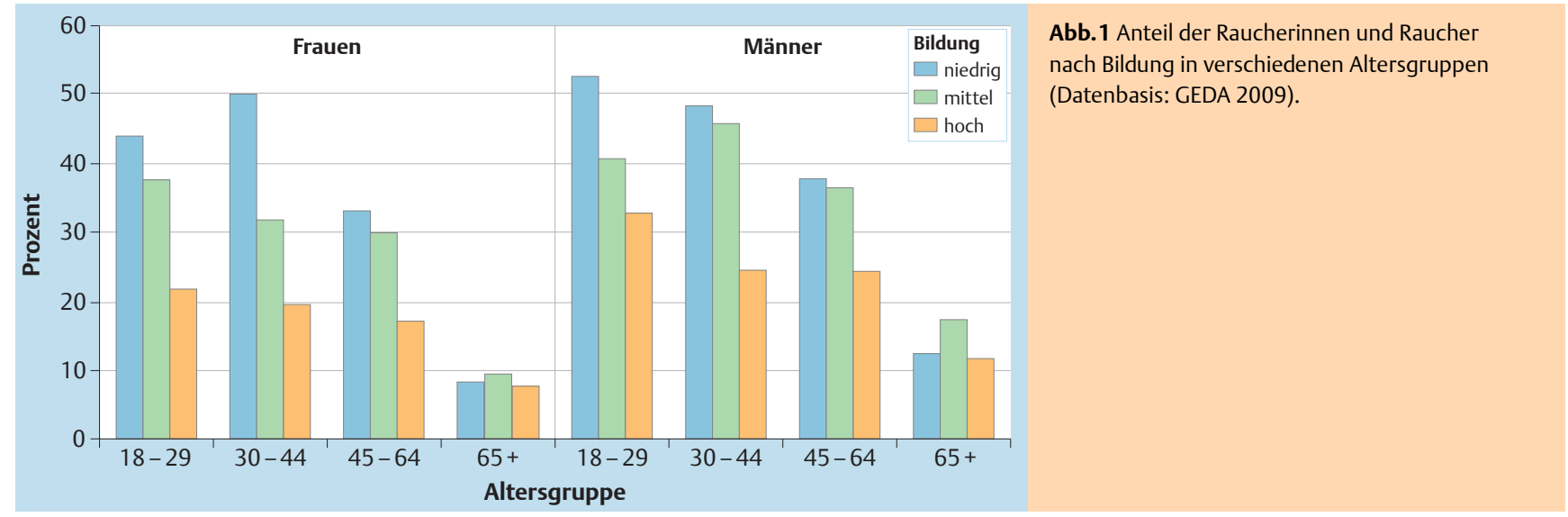

In Deutschland rauchen etwa 27\% der Bevölkerung, etwas mehr Männer als Frauen, wobei sich dieser Trend bei den Jugendlichen bereits umgekehrt hat. Zudem korreliert die Raucherquote invers mit dem Einkommen [9] ( Abb.1). In Deutschland sterben jährlich 110 000-140 000 Menschen an den Folgen des Rauchens [41]. Ein Rauchstopp ist mit einer „number needed to treat" von 2 (längerfristig betrachtet) eine der wirksamsten bekannten medizinischen Maßnahmen [25]. Tab.1 zeigt, dass zwischen 2003 und 2009 - einer Zeit intensiver Aufklärung über die Gefahren des Rauchens - der Prozentsatz der Raucher an der Gesamtbevölkerung abgenommen hat. Ausnahmen bildeten Frauen über 45 und Männer zwischen 45 und 64 Jahren. Immerhin kam es auch in diesen Gruppen zu einem leichten Rückgang des Anteils starker Raucher, der in den meisten anderen Subgruppen sogar sehr deutlich ausfiel.

\section{kurzgefasst}

Rauchen macht Menschen krank und verkürzt ihr Leben. Dieser Schadstoff könnte prinzipiell komplett „ausgeschaltet“ werden. Der Hausarzt hat bei der Entwöhnung die Schlüsselrolle: Schon ein kurzes Gespräch erzielt nachweisliche Effekte, nachhaltige und empathische Motivation führt zu vermehrter Nikotinkarenz.

\section{Diagnostik}

$\nabla$

Nicht jedem Raucher gelingt ein Rauchausstieg im Selbstversuch, trotz vorhandener Ausstiegsbereitschaft. Ungefähr die Hälfte der regelmäßigen Raucher zeigt eine Tabakabhängigkeit im Sinne der ICD-10-Kriterien [4, 18]. Um die biopsychosozialen Komponenten der Tabakabhängigkeit (ICD-10, WHO) [18] zu erfassen, ist eine Anamnese $[3,16]$ erforderlich, die neben einer kategorialen und einer dimensionalen Einordnung weitere Hintergrundinformationen über das Rauchverhalten beinhaltet. So gelingt es, einen passgenauen Einstieg in die Motivationsarbeit und Therapieplanung zu finden.

\section{Kategoriale Diagnostik nach ICD-10 [6]}

- Starkes Verlangen bzw. eine Art Zwang, Tabak zu konsumieren

- Verminderte Kontrollfähigkeit bzgl. Beginn, Beendigung oder Menge des Konsums

- Körperliches Entzugssyndrom bei Beendigung oder Reduktion des Konsums

- Toleranzentwicklung bzgl. der Wirkung der Substanz

- Anhaltender Tabakkonsum trotz Kenntnis der eindeutig schädlichen Folgen

- Aufgabe oder Vernachlässigung anderer wichtiger Interessen wegen des Tabakkonsums

Tab.1 Veränderung des Anteils der Raucherinnen und Raucher bzw. der starken Raucherinnen und Raucher in verschiedenen Altersgruppen; Datenbasis: Gesundheitssurvey 2003, GEDA 2009.

\begin{tabular}{|c|c|c|c|c|c|c|c|c|}
\hline & \multicolumn{4}{|c|}{ Rauchen } & \multicolumn{4}{|c|}{ Starkes Rauchen } \\
\hline & 2003 & 2009 & Differenz & p-Wert & 2003 & 2009 & Differenz & p-Wert \\
\hline \multicolumn{9}{|l|}{ Frauen } \\
\hline 18 bis 29 Jahre & $46,2 \%$ & $37,9 \%$ & $-8,3$ & $p<0,010$ & $6,3 \%$ & $3,7 \%$ & $-2,6$ & $p<0,050$ \\
\hline 30 bis 44 Jahre & $39,6 \%$ & $33,4 \%$ & $-6,2$ & $\mathrm{p}<0,010$ & $12,8 \%$ & $6,8 \%$ & $-6,0$ & $p<0,001$ \\
\hline 45 bis 64 Jahre & $27,8 \%$ & $29,4 \%$ & $+1,6$ & $p=0,277$ & $8,5 \%$ & $7,0 \%$ & $-1,5$ & $p=0,114$ \\
\hline 65 Jahre und älter & $7,0 \%$ & $8,7 \%$ & $+1,7$ & $p=0,204$ & $0,9 \%$ & $0,7 \%$ & $-0,2$ & $p=0,562$ \\
\hline Gesamt & $28,9 \%$ & $26,1 \%$ & $-2,8$ & $p<0,050$ & $7,4 \%$ & $4,7 \%$ & $-2,7$ & $p<0,001$ \\
\hline \multicolumn{9}{|l|}{ Männer } \\
\hline 18 bis 29 Jahre & $54,5 \%$ & $43,2 \%$ & $-11,3$ & $p<0,010$ & $12,4 \%$ & $5,2 \%$ & $-7,2$ & $p<0,001$ \\
\hline 30 bis 44 Jahre & $45,9 \%$ & $42,2 \%$ & $-3,7$ & $p=0,068$ & $18,3 \%$ & $12,4 \%$ & $-5,9$ & $p<0,010$ \\
\hline 45 bis 64 Jahre & $33,4 \%$ & $34,6 \%$ & $+1,2$ & $p=0,526$ & $13,2 \%$ & $12,1 \%$ & $-1,1$ & $p=0,677$ \\
\hline 65 Jahre und älter & $17,1 \%$ & $13,7 \%$ & $-3,4$ & $p=0,112$ & $4,7 \%$ & $2,4 \%$ & $-2,3$ & $\mathrm{p}<0,050$ \\
\hline Gesamt & $38,3 \%$ & $33,9 \%$ & $-4,4$ & $p<0,001$ & $13,2 \%$ & $9,2 \%$ & $-4,0$ & $p<0,001$ \\
\hline
\end{tabular}


Von diesen Kriterien müssen zeitgleich mindestens 3 innerhalb des letzten Jahres aufgetreten sein, um von „Tabakabhängigkeit“ zu sprechen.

Beim Nikotinentzugssyndrom müssen nach ICD-10 mindestens 2 der folgenden Kriterien zutreffen [6]:

- Starkes Verlangen nach Nikotin

- Krankheitsgefühl oder Schwäche

- Angst

- Dysphorische oder gereizte Stimmung

- Innere Unruhe

- Insomnie

- Appetitsteigerung

- Vermehrter Husten

- Konzentrationsstörungen

- Ulzerationen der Mundschleimhaut

\section{Dimensionale Diagnostik}

Der Fagerströmtest für Nikotinabhängigkeit (FTND) [22, 25] erfasst die Intensität des Rauchverlangens und somit den Schweregrad der Abhängigkeit. Die Intensität ist ein aussagekräftiger Prädiktor von mittel- bis langfristiger Abstinenz. Der FTND umfasst 6 Fragen und ist ein durch seine Kürze beliebtes Instrument, das dennoch substanzielle Informationen liefert. Psychologische und medikamentöse Therapieempfehlungen richten sich nach dem FTND-Wert [6].

\section{Bestimmung der Schadstoffbelastung und Rauchintensi-} tät

Die Messung der CO-Konzentration in der Atemluft gibt Aufschluss über die Intensität des Tabakkonsums. Bei einem Konsum von über 25 Zigaretten/Tag werden CO-Werte von > 40 ppm erreicht. Ein CO-Wert von $<5$ ppm gilt als Abstinenzbeleg bzw. als Nachweis, dass der letzte Konsum vor mehr als 8 Stunden stattgefunden hat. Die Messung kann ein Motivationsfaktor bei der Entwöhnung sein. Weitere biochemische Parameter sind Nikotin-Plasma-Konzentration, Thiocyanat-Konzentration, Cotinin-Spiegel im Serum, Speichel und Urin [6].

\section{Rauchanamnese}

Neben der Diagnose und Stärke der Abhängigkeit besteht die Möglichkeit, weitere Hintergrundinformationen durch leitlinienbasierte Dokumentationsbögen [4] zu erfassen. Hierzu zählen Einstiegsalter, Dauer der Raucherkarriere, aktuelles Rauchverhalten, Ressourcen, bisherige Aufhörversuche, medizinische Faktoren, aktuelle Bereitschaft zur Beendigung des Rauchens und Informationen zur sozialen Unterstützung für die Abstinenz. Eine Erhebung der Packungsjahre (pack-years = täglich konsumierte Zigarettenpackungen $\times$ Zahl der Raucherjahre) gibt Aufschluss über das Risiko tabakassoziierter Folgeerkrankungen.

\section{kurzgefasst}

Einer Erfolg versprechenden Raucherentwöhnung geht eine exakte Diagnostik voraus, um Motivationsstrategien und Therapie der aktuellen Situation des Patienten individuell anzupassen.

\section{Kognitive Verhaltenstherapie zur Bewältigung} der Nikotin-/Tabakabhängigkeit $\checkmark$

„Gesagt ist noch nicht gehört“.

„Gehört ist noch nicht verstanden“.

„Verstanden ist noch nicht einverstanden“.

„Einverstanden ist noch nicht angewendet“.

„Angewendet ist noch nicht beibehalten“.

(Konrad Lorenz)

Das Zitat fasst prägnant zusammen, was das Phasenmodell der Verhaltensänderung (Transtheoretisches Modell nach Prochaska und DiClemente) [32] besagt: den Raucher dort abzuholen, wo er gerade im Prozess der Verhaltensänderung steht. Am Anfang der Tabakentwöhnung steht daher die Standortbestimmung. Nach diesem Modell ist das Aufhören kein singuläres Ereignis, sondern ein dynamischer Veränderungsprozess, zu dem auch gelegentliche Rückschritte in eine vorherige Phase gehören. Er durchläuft folgende Phasen:

\section{Absichtslosigkeit}

Der Raucher hat bisher noch nicht in Erwägung gezogen, mit dem Rauchen aufzuhören.

\section{Absichtsbildung}

Der Raucher hat ernsthaft in Erwägung gezogen, mit dem Rauchen aufzuhören.

3.Vorbereitung

Der Raucher hat die Absicht, in naher Zukunft das Rauchen aufzugeben.

4.Handlung

Der Raucher hat einen Rauchstopp unternommen.

5.Aufrechterhaltung

Der Raucher will die Karenz aufrecht erhalten und nicht wieder mit dem Rauchen anfangen.

6.Abschluss

Der Raucher hat einen stabilen Zustand des Nichtrauchens erreicht.

Interventionen richten sich nach dem Stadium des Veränderungsprozesses zur Bewältigung der Nikotinabhängigkeit.

\section{Stadium der Absichtslosigkeit}

Raucher sind nicht willens aufzuhören. Sie bewerten die Nachteile des Aufhörens höher als die Vorteile oder die eigenen Erfolgschancen als äußerst gering. In dieser Phase sind handlungsorientierte Raucherentwöhnungsprogramme noch verfrüht. Vielmehr können die Raucher von Informationen zum Thema profitieren: Gefahren des Rauchens, persönlicher Gewinn durch Rauchfreiheit, Möglichkeiten, erfolgreich aufzuhören, ggf. auch von Kontakten mit erfolgreichen Ex-Rauchern (Modell-Lernen).

Im Stadium der Absichtslosigkeit zielen motivierende Gespräche vor allen Dingen darauf ab, den Entwöhnungswillen zu steigern. Die Gespräche sollten die „fünf R“ zur Motivationssteigerung berücksichtigen [34]:

- Relevanz: persönlichen Bezug zur Rauchfreiheit herausstellen,

- Risiken des fortgesetzten Zigarettenrauchens benennen,

- Reize: persönliche Vorteile der Tabakabstinenz deutlich machen,

- Riegel: Hindernisse für eine Verhaltensänderung aufspüren und beseitigen,

- Repetition: nicht aufhörbereite Raucher bei jedem Kontakt auf die Notwendigkeit und Sinnhaftigkeit des Rauchstopps ansprechen. 
Einer kleinen finnischen Studie zufolge [31] befinden sich mehr als 50\% der Raucher in diesem Stadium der Absichtslosigkeit. Für diese große Zahl können vor allen Dingen internetbasierte, also populationsbezogene Behandlungsangebote hilfreich sein.

\section{Stadium der Absichtsbildung}

Im Stadium der Absichtsbildung ist der Raucher noch ambivalent, ob er wirklich mit dem Rauchen aufhören will, er zieht es jedoch in Erwägung. Raucher können sich in dieser Phase durchaus jahrelang bewegen. Sie profitieren von erfolgreichen Modellen, d.h. vom Kontakt zu Ex-Rauchern. Ausschlaggebend können sich darüber hinaus unerwartete Lebensereignisse wie Herzinfarkt oder Lungenkrebsdiagnose im Bekanntenkreis auswirken. Eine Möglichkeit, den Patienten anzuregen, Bilanz zu ziehen und persönliche Werte (wie Gesundheit) dem eigenen Verhalten (Rauchen) gegenüber zu stellen.

Eine kognitiv-verhaltenstherapeutische Analyse kann mit dem Raucher erarbeiten, in welchen kritischen Lebenssituationen das Rauchen hilfreich ist:

- Wird das Rauchen zur Stressbewältigung im Beruf eingesetzt?

- Hilft es, eine innere Leere zu überbrücken?

- Kann ein alternatives Verhalten an die Stelle der vermeintlichen Problembewältigung treten?

- Es ist hilfreich, Ängste und Bedenken anzusprechen, beispielsweise Gewichtszunahme und Entzugserscheinungen.

\section{Stadium der Vorbereitung}

In diesem Stadium ziehen Raucher einen Rauchstopp innerhalb der nächsten vier Wochen in Erwägung und sind bereits von den Vorteilen überzeugt. Unter Umständen fürchten sie sich vor Rückfällen und suchen nach geeigneten professionellen Maßnahmen bzw. nach ärztlicher Unterstützung beim Selbstversuch. Klassische Nichtrauchertrainings werden hauptsächlich von Rauchern in dieser Phase wahrgenommen, in der sich jedoch nur ein Fünftel aller Raucher befindet [36].

Beispiele für professionelle Angebote in der Tabakentwöhnung sind Nichtraucher in 6 Wochen (Universitätsklinik für Psychiatrie und Psychotherapie, Tübingen), Das Rauchfrei-Programm (IFT München), Rauchfrei Werden (IRT München), Curriculum Tabakentwöhnung (Bundesärztekammer).

\section{Stadium der Handlung}

Am erfolgreichsten ist die Punkt-Schluss-Methode im Vergleich zur schrittweisen Reduktion des Zigarettenkonsums. In der Handlungsphase geht es darum, die etablierten kognitiv-verhaltenstherapeutischen Veränderungstechniken in Gruppen- oder Einzeltherapie zur Vorbereitung und Durchführung des Ausstiegs einzusetzen. Auch ergänzende Methoden (Hypnose, Akupunktur) können zum Einsatz kommen. Hilfreich ist oft eine pharmakologische Unterstützung (Nikotinsubstitution, psychopharmakologische Therapie). In dieser Phase besteht eine große Rückfallgefahr, für die gut ausgearbeitete Rückfallpräventionsstrategien existieren [10].

Da etwa zwei Drittel aller Raucher in den ersten sechs Monaten rückfällig werden, sollten sie in dieser Zeit begleitet werden und an Auffrischsitzungen teilnehmen, die „Rückfallprophylaxe“ aktiv zum Thema machen. Problemlösefertigkeiten können aufgebaut, die kognitiven Voraussetzungen verbessert und die möglichen Versuchungssituationen antizipiert werden. Eine Verstärkung der neu erworbenen Rauchfreiheit in Form von Selbstbe- lohnung oder Anerkennung durch Therapeuten und Angehörige stützt die Veränderung.

\section{Stadium der Aufrechterhaltung}

Nach erfolgreicher Tabakkarenz beginnt das Stadium, in dem es um die Aufrechterhaltung des Rauchstopps geht. Prochaska zufolge dauert dieses Stadium bis zu fünf Jahre [32]. In dieser Zeit braucht der Ex-Raucher die Möglichkeit zum Kontakt mit psychologischen Beratungsstellen bei kritischen Lebensereignissen und großer Rückfallgefahr. Die Raucherentwöhnung sollte in ein Nachsorgeprogramm eingebettet sein (z.B. Bundeszentrale für gesundheitliche Aufklärung 01805-313131; Rauchertelefon des Deutschen Krebsforschungszentrums 06221-424200; „HelpLine-Bayern“ 0800-1418141).

Günstige Prädiktoren für dauerhafte Tabakabstinenz sind höheres Alter, geringere Stärke der körperlichen Abhängigkeit, hohe Selbstwirksamkeitserwartung bzw. hohe Abstinenzzuversicht und soziale Unterstützung. Gelingt es dem Raucher, zwei Phasen der Veränderung innerhalb eines Monats zu durchschreiten, so vervierfacht sich die Erfolgswahrscheinlichkeit. Wo möglich, sollten der nichtrauchende Partner und/oder andere soziale Bezugsgruppen zur Unterstützung des dauerhaften Nichtrauchens einbezogen werden.

\section{Abschlussstadium}

Es ist dem Ex-Raucher endgültig gelungen, nicht mehr mit dem Rauchen anzufangen. Auch schwierige Versuchungssituationen führen nicht mehr zum Rückfall.

Interventionsprogramme sollen den Raucher von einem zum nächsten Stadium unterstützend begleiten und damit Schritt für Schritt einer dauerhaften Tabakkarenz näher bringen. Bereits der Wechsel in ein höheres Stadium ist ein Erfolg. Professionellen Angeboten in komprimierter Form gelingt es aber nicht selten, diesen Entwicklungsprozess innerhalb einer Woche bis zum 4./5. Stadium voranzubringen.

kurzgefasst

Die Raucherentwöhnung umfasst einen Prozess von bis zu 5 Jahren. Eine erfolgreiche kognitiv-verhaltenstherapeutische Intervention analysiert, in welchem der sechs Stadien des Veränderungsprozesses sich der Raucher befindet und welche Intervention hierfür hilfreich sein kann. Bereits der Wechsel in eine höhere Phase ist ein Erfolg.

\section{Medikamentöse und nicht-medikamentöse Unterstützung in der Tabakentwöhnung}

Beratung alleine ist nicht so effektiv wie in Kombination mit medikamentöser Unterstützung. Deshalb können beide Maßnahmen angemessen kombiniert werden. Den größten Stellenwert in der medikamentösen Therapie haben derzeit nikotinhaltige Präparate sowie Vareniclin und Bupropion [7]. Die deutschen und amerikanischen Leitlinien empfehlen eine medikamentöse Begleittherapie.

\section{Nikotinersatztherapie}

Die Nikotinersatztherapie erwies sich in insgesamt 111 Studien ( $\mathrm{n}=40000)$ als wirksam [35]. Nikotin ist nicht für die gesundheitlichen Schäden des Tabakkonsums verantwortlich. Aus diesem Grund kann es bei angemessener, am bisherigen Konsum 
orientierter Dosierung ohne Gefahr für 2-3 Monate verordnet werden. Ziel ist eine vollständige Entwöhnung durch langsame Dosisreduktion.

Nikotinpflaster Die Pflaster geben Nikotin kontinuierlich ab und lindern so Entzugssymptome. Die Wirklatenz beträgt ca. 2 Stunden. Es gibt Präparate mit 16 und 24 Stunden Wirksamkeit. Ab 20 Zigaretten/Tag wird meist die höchste Dosierung für 4-8 Wochen verordnet, die weitere Reduktion erfolgt in 2 Stufen mit je 2-wöchigem Abstand. In ausgewählten Fällen ist eine Verlängerung der Therapie bis zu 6 Monaten bei täglichem Wechsel der Klebestelle möglich [23]. Eine Kontraindikation ist Pflasterallergie. Nikotinpflaster sind mit Nikotinkaugummis gut kombinierbar.

Nikotinkaugummi Der Nikotinkaugummi ermöglicht eine individuelle und bedarfsgerechte Nikotinzufuhr. So wird sowohl die Entzugssymptomatik gelindert als auch Suchtdruck vorgebeugt. Die maximale Wirkung tritt nach 20-30 min ein. Empfohlen wird eine Gabe nach Plan, um eine Abhängigkeitsentwicklung zu vermeiden. Es gibt Dosierungen von 2 und $4 \mathrm{mg}$. Ab 20 Zigaretten/Tag werden $4 \mathrm{mg}$ empfohlen. Der Tagesbedarf sollte langsam reduziert werden, Ziel ist ein Absetzen nach 2-3 Monaten. Mögliche Nebenwirkungen sind Schleimhautirritationen und Magenbeschwerden [23].

Nikotintabletten Sublingualtabletten zergehen unter der Zunge innerhalb von 10-20 min, das Nikotin wird über die Mundschleimhaut aufgenommen. Die maximale Nikotinkonzentration wird nach ca. 20-30 min erreicht. Nebenwirkungen: siehe Nikotinkaugummi.

Nikotinnasalspray Die Anwendung ermöglicht hoch dosierte Nikotingaben über die Nasenschleimhaut. Das Wirkmaximum wird nach wenigen Minuten erreicht. Das Spray ist vor allem bei stark abhängigen Rauchern mit einem Fagerström-Score von mindestens 7 Punkten effektiv. Die durch die rasche Anflutung bedingte Gefahr einer Abhängigkeitsentwicklung kann durch konsequente Reduktion der Tagesdosis minimiert werden. Eine mögliche Nebenwirkung ist die schmerzhafte Gefäßkontraktion der Nasenschleimhaut. Unter den Nikotinersatzprodukten ist Nasenspray am effektivsten, kann aber in Deutschland nur über internationale Apotheken bezogen werden.

Nicorette Inhaler Im Mundstück des Inhalators befindet sich eine Kapsel, die bei Inhalation Nikotin freisetzt. Der Inhalator imitiert in seiner Handhabung die Zigarette und wird daher von Suchttherapeuten kritisch beurteilt. Als Nebenwirkung kann eine Schleimhautirritation auftreten.

\section{Vareniclin}

Vareniclin ist seit 2007 für die Tabakentwöhnung zugelassen. Direkte Stimulation des spezifischen Nikotinrezeptors kann Verlangen und die Entzugssymptomatik dämpfen. Das Rauchen einer Zigarette führt so zu keinem befriedigenden Effekt, da das Nikotin keine zusätzliche Wirkung am Rezeptor entfalten kann. Vareniclin wird in den ersten 7 Tagen einschleichend dosiert, um das Auftreten von Übelkeit zu vermeiden. Der Rauchstopp soll spätestens am 8 . Tag erfolgen. Ab dem 8 . Tag wird $2 \times 1 \mathrm{mg}$ täglich als Enddosis für weitere 11 Wochen eingenommen. Bei hoher Rückfallgefahr kann Vareniclin für weitere 12 Wochen rezeptiert werden.
Vareniclin ist gemäß 7 Studien erfolgreicher als Placebo und eventuell wirksamer als Nikotinersatz (1 Studie) und Bupropion (3 Studien) [5, 13]. Mögliche Nebenwirkungen sind Schwindel, Übelkeit und Depressionen. Die Zulassungsbehörden haben wegen Suizidalität und kardialen Komplikationen vom Einsatz bei psychisch auffälligen oder kardial schwer erkrankten Patienten abgeraten [13]. Vareniclin ist rezeptpflichtig, jedoch nicht erstattungsfähig.

\section{Bupropion}

Bupropion ist ein atypisches Antidepressivum, das die Wiederaufnahme von Dopamin und Noradrenalin hemmt. Seit 2000 ist es zur Tabakentwöhnung in Deutschland zugelassen. In der 1. Therapiewoche wird morgens $1 \times 150 \mathrm{mg}$, in der 2 . Woche werden $2 \times 150 \mathrm{mg}$ (morgens und nachmittags) verabreicht. Die Therapie dauert i.d.R. 8 Wochen und kann bei schwerer Entzugssymptomatik verlängert werden. Bupropion erhöht die langfristigen Abstinenzquoten [26], es ist ähnlich wirksam wie die Nikotinersatztherapie. Mögliche Nebenwirkungen sind Tremor, Schlafstörungen, Mundtrockenheit und Schwindel. Bei höherer Dosierung können Krampfanfälle mit einer Häufigkeit von 0,1\% auftreten. Bupropion ist rezeptpflichtig, jedoch nicht erstattungsfähig.

\section{Akupunktur}

Akupunktur wird bei der Tabakentwöhnung häufig eingesetzt, obwohl bisher kein Effekt nachweisbar ist [37] und (inter)nationale Leitlinien sie nicht empfehlen [7, 23].

\section{Hypnose}

Hypnose kann die Verhaltenstherapie unterstützen. Der Erfolg ist oft nur kurzfristig, kontrollierte Studien stehen noch aus [14]. Ergebnisse einer Studie der Universitätsklinik Tübingen und des Universitätsklinikums Hamburg-Eppendorf werden 2013 erwartet [5].

\section{kurzgefasst}

\section{Medikamente und Nikotinersatztherapie können bei der} Raucherentwöhnung unterstützend eingesetzt werden.

\section{Konsequenz für Klink und Praxis}

- Rauchen ist eine der bedeutendsten vermeidbaren Ursachen von Erkrankung und vorzeitigem Tod, schädigt viele Organsysteme und schränkt damit auf längere Sicht die Lebensqualität stark ein.

- Bei der Motivation zur Tabakentwöhnung und Aufrechterhaltung der Abstinenz kommt dem Hausarzt eine Schlüsselrolle zu. Bereits der empathisch formulierte ärztliche Rat, das Rauchen aufzugeben, hat hohe Wirksamkeit.

- Bei der hausärztlichen Betreuung von Rauchern sollte das Thema immer wieder angesprochen und der Patient im Motivationsprozess an dem Punkt „abgeholt“ werden, an dem er sich gerade befindet.

- Die Raucherentwöhnung ist ein stufenweiser Prozess, der kognitiv-verhaltenstherapeutisch und auch medikamentös erfolgreich unterstützt werden kann. 
Autorenerklärung: Die Autoren erklären, dass sie keine finanzielle Verbindung mit einer Firma haben, deren Produkt in diesem Beitrag eine Rolle spielt (oder mit einer Firma, die ein Konkurrenzprodukt vertreibt).

Literatur

1 Andreas $S$ et al. Tabakentwöhnung bei COPD. Pneumologie 2008; 62 255-272

2 Andreas $S$ et al. Klinische Leitlinie Tabakentwöhnung bei chronisch obstruktiver Lungenerkrankung: Eine effektive und sinnvolle medizinische Intervention. Dtsch Arztebl 2009; 106: 276-282

3 Arzneimittelkommission der deutschen Ärzteschaft. Empfehlungen zur Therapie der Tabakabhängigkeit. 37.: Sonderheft 2 Arzneiverordnung in der Praxis Band 2010; http://www.akdae.de/Arzneimitteltherapie/TE/A-Z/PDF/Tabakabhaengigkeit.pdf (letzter Zugriff 14.3.2013)

4 Batra A, Buchkremer G. Raucherentwöhnung. In: Meinlschmidt G, Schneider S, Margraf J Hrsg. Lehrbuch der Verhaltenstherapie. Band 4 Materialien für die Psychotherapie Berlin, Springer 2012; 371-382

5 Batra A, Lindinger P. Curriculum Tabakabhängigkeit und Entwöhnung. Kursprogramm „Nichtraucher in 6 Wochen“. Tübingen, Universitätsklinik für Psychiatrie und Psychotherapie 2012

6 Batra A, Peukert P. Biologische Marker und Diagnostik der Nikotin- und Tabakabhängigkeit. In: Singer MV, Batra A, Mann K Hrsg. Alkohol und Tabak. Grundlagen und Folgeerkrankungen. Stuttgart, New York, Thieme 2011; 526-529

7 Batra A, Schütz CG, Lindinger P. Tabakabhängigkeit. In: Schmidt LG, Gastpar $M$, Falkai $P$, Gaebel $W$ Hrsg. Evidenzbasierte Suchtmedizin. Behandlungsleitlinie Substanzbezogene Störungen. Köln, Deutscher Ärzte-Verlag 2006; 91-142

8 Batra A, Lindinger P. Tabak. In: Batra A, Bilke-Hentsch $O$ Hrsg. Praxisbuch Sucht. Therapie der Suchterkrankungen im Jugend- und Erwachsenenalter. Stuttgart, New York, Thieme 2012; 100-127

9 Becher $H$ et al. Passivrauchen - ein unterschätztes Gesundheitsrisiko. Rote Reihe Tabakprävention und Tabakkontrolle Band 5. 2. Auflage. Heidelberg, Deutsches Krebsforschungszentrum 2006; http://www.dkfz.de/de/tabakkontrolle/download/Publikationen/RoteReihe/Passivrauchen_Band_5_2Auflage.pdf (letzter Zugriff 14.3.2013)

10 Buchkremer G. Raucherentwöhnung. Psychologische und pharmakologische Methoden. Stuttgart, Thieme 1989

11 Bühler A et al. Literaturauswertung zur Wirksamkeit von Warnhinweisen auf Zigarettenpackungen. http://www.ift.de/literaturverzeichnis/Bd_166_Zig_Warnhinweise.pdf (letzter Zugriff 14.3.2013)

12 Bundeszentrale für gesundheitliche Aufklärung (BZgA), Bundesärztekammer Hrsg. Leitfaden zur Kurzintervention bei Raucherinnen und Rauchern. Köln, 2006

13 Cahill K, Steak LF, Lancaster T. Nicotine receptor partial agonists for smoking cessation. Cochrane Database Syst Rev 2012; CD006103

14 Cochrane review of hypnosis for smoking cessation. The Cochrane Library http://www.hypnosisandsuggestion.org/cochrane-review-of-hypnosis-for-smoking-cessation.html (letzter Zugriff 14.3.2013)

15 Deutsche Gesellschaft für Pneumologie und Beatmungsmedizin. Tabakentwöhnung bei COPD. Pneumologie 2008; 62: 255-272

16 DGPPN/DG Sucht. Leitlinien zur Behandlung des Rauchens. http://www.sucht.de/tl_files/pdf/11_05_03.pdf (letzter Zugriff 14.3.2013)

17 Die Welt. Eine-Milliarde-Raucher-Tote-in-diesem-Jahrhundert. http://www.welt.de/wissenschaft/article991427/Eine-Milliarde-RaucherTote-in-diesem-Jahrhundert.html (letzter Zugriff 14.3.2013)

18 Dilling H, Mombour W, Schmidt MH Hrsg. Internationale Klassifikation psychischer Störungen. ICD-10 Kapitel V (F). Klinisch-diagnostische Leitlinien 7.: revidierte Fassung. Bern, Huber 2010
19 Dong CY, McRobbie H, Walker N et al. Hypnotherapy for smoking cessation. Cochrane Database Syst Rev 2010; CD001008

20 Etzel M, Mons U, Schmitt S et al. Raucherentwöhnung in Deutschland 2007 - Struktur der ambulanten Therapieangebote zur Tabakentwöhnung und Raucherberatung. Bundesgesundheitsbl - Gesundheitsforsch - Gesundheitsschutz 2008; 51: 1453-1461

21 Ezzati M, Lopez AD. Estimates of global mortality attributable to smoking in 2000. Lancet 2003; 362: 847-852

22 Fagerstrom $\mathrm{KO}$, Schneider NG. Measuring nicotine dependence: a review of the Fagerstrom Tolerance Questionnaire. J Behav Med 1989; 23: 159182

23 Fiore MC, Jaén CR, Baker TB et al. Treating Tobacco Use and Dependence: 2008 Update. Quick Reference Guide for Clinicians Rockville, MD, U.S. Department of Health and Human Services. Public Health Service April 2009; http://guideline.gov/content.aspx?id=12520 (letzter Zugriff 14.3.2013)

24 Focus online. 3300 Tote durch Passivrauchen. 08.12.2005; http://www.focus.de/gesundheit/gesundleben/nichtrauchen/news/tabakopfer_aid_102351.html (letzter Zugriff 14.3.2013)

25 Heatherton TF, Kozlowski LT, Frecker RC et al. The Fagerström Test for Nicotine Dependence: a revision of the Fagerström Tolerance Questionnaire. Br J Addict 1991; 86: 1119-1127

26 Hughes JR, Stead LF, Lancaster T. Antidepressants for smoking cessation. Cochrane Database Syst Rev 2007; CD000031

27 Keil $U$. Coronary artery disease: The role of lipids, hypertension and smoking. Basic Res Cardiol 2000; 95 (Suppl. 01): 52-58

28 Krebs H. 20 Sekunden zum Nachdenken. Eine Präventionsbroschüre der Krebsliga Schweiz. 4. überarbeitete Auflage. Bern, 2011

29 Mackay J, Eriksen M. The Tobacco Atlas.World Health Organization 2002; http://www.who.int/tobacco/media/en/title.pdf (letzter Zugriff 14.3.2013)

30 Morrow M, Brands A. Fact Sheet on Gender, Health and Tobacco. Geneva, Switzerland., WHO Tobacco Free Initiative October 2003; http://whqlibdoc.who.int/gender/2003/a85587.pdf (letzter Zugriff 14.3.2013)

31 Pallonen UE, Fava JL, Salonen JT et al. Readiness for smoking-change among middle-aged Finnish men. Addict Behav 1992; 17: 415-423

32 Prochaska JO, DiClemente CC. Transtheoretical therapy: Toward a more integrative model of change. Psychotherapy 1982; 19: 276-278

33 Singer MV, Batra A, Mann K Hrsg. Alkohol und Tabak. Grundlagen und Folgeerkrankungen. Stuttgart, Thieme 2011

34 Soria R, Legido A, Escolano C et al. A randomised controlled trial of motivational interviewing for smoking cessation. Br J Gen Pract 2006; 56: 768-774

35 Stead LF, Bergson G, Lancaster T. Physician advice for smoking cessation. Cochrane Database Syst Rev 2008; CD000165

36 Unland $H$. Wir gewöhnen uns das Rauchen ab - wieder frei und selbstbestimmt leben - ein kognitiv-verhaltenstherapeutisches Raucherentwöhnungsprogramm Material Nr. 28; Tübingen, dgvt-Verlag 1995

37 White AR, Rampes H, Liu JP et al. Acupuncture and related interventions for smoking cessation. Cochrane Database Syst Rev 2011; CD000009

38 WHO Tobacco Free Initiative, WHO Noncommunicable Disease and Mental Health. Why tobacco is a public health priority. http://www.who.int/tobacco/health_priority/en/ (letzter Zugriff 14.3.2013)

39 Zeit online (AFP/Reuters). Durch Passivrauchen sterben jährlich 600.000 Menschen; http://www.zeit.de/wissen/gesundheit/2010-11/passivrauchen-who-kinder (letzter Zugriff 14.3.2013)

40 Zylka-Menhorn V. Rauchen: Suchtmerkmale bewiesen. Dtsch Arztebl 2008; 105: A-1420 / B-1228 / C-1196

41 Deutsche Hauptstelle für Suchtfragen e.V.. Tabak. http://www.dhs.de/datenfakten/tabak.html (letzter Zugriff 14.3.2013) 